\title{
On Semi-Positivity and Filtered Frobenius Crystals
}

\author{
By
}

\section{Shinichi MochIZUKI*}

\section{§0. Introduction}

The purpose of this paper is to prove that certain subquotients of the $\mathscr{M} \mathscr{F}^{\nabla}$-objects of Faltings ([1]) are semi-positive. In particular, we obtain an algebraic proof of a result (generalizing those of [5], [9]) on the semi-positivity of the higher direct images of certain kinds of sheaves for semistable families of algebraic varieties. Our Main Theorem, proven in $\S 3$, is as follows:

Theorem 3.4. Let $f:(X, E) \rightarrow(S, D)$ be a semistable family of varieties of relative dimension $d$, with $S$ a smooth, proper scheme over a field $L$ of characteristic zero. Let $\left(\mathscr{A}, \nabla_{\mathscr{A}}, F^{i}(\mathscr{A})\right)$ be a globally crystalline filtered vector bundle with connection on $(X, E)$. Then for any nonnegative integer $\alpha$, the coherent sheaf of $\mathcal{O}_{\mathbf{S}}$-modules

$$
\mathbb{R}^{\alpha} f_{*}\left(\omega_{X / S} \otimes_{\mathcal{O}_{X}}\left(\mathscr{A} / F^{1}(\mathscr{A})\right)^{\vee}\right)
$$

is a semi-positive vector bundle, as are all of its tensor powers.

(The term "semistable" (respectively, "globally crystalline") is defined in $\S 1$ (respectively, §3).) In particular, this Theorem implies the following:

Corollary 3.5. Let $f:(X, E) \rightarrow(S, D)$ be as above. Then for any nonnegative integer $\alpha$, the coherent sheaf of $\mathcal{O}_{S}$-modules

$$
f_{*}\left(\left(\Omega_{X \mid S}^{\log }\right)^{\alpha}\right)
$$

is a semi-positive vector bundle, as are all of its tensor powers.

Thus, Fujita and Kawamata proved the above Corollary in the case $\alpha=d$.

Roughly speaking, the idea of the proof is as follows. We restrict to a

Received February 24, 1994. Revised July 20, 1994.

1991 Mathematics Subject Classifications: 14F30.

* Research Institute for Mathematical Sciences, Kyoto University, Kyoto 606, Japan. 
curve, and consider the possibility of quotients of the bundle in question that have negative degree. If such a quotient did exist, it would mean that we could construct a large number of sections of a space of bounded dimension. This concludes the proof.

Since the proof is substantially simpler and less high-powered in the case when the fibres of $f$, as well as the base $S$, are one-dimensional and the filtered vector bundle is trivial, we present the proof in that special case in an Appendix (which is logically independent of the rest of the paper, except for basic facts and definitions given in $\S 1$ ). We believe that this special case sheds light on the general case. In essence, what makes the proof easier in this case is the fact that we have a very physical and concrete realization of 1-motives and Hodge theory (for trivial coefficients), namely, the theory of semiabelian schemes and the Picard group. It is the lack of such explicit tools in the higher-dimensional case with nontrivial coefficients that requires one, if one is to carry out an analogous proof, to employ more abstract tools.

In [7], Illusie has proven similar results using essentially equivalent techniques to our own, although from a somewhat different point of view. Namely, Illusie works modulo $p$, and obtains the following result:

Theorem (given as Remark 4.18 in [7]). If $f:(X, E) \rightarrow(S, D)$ is as in Theorem 3.4, and $\mathscr{L}$ is an ample line bundle on $S$, then for all $i \geq 1, j \geq 0$, we have:

$$
H^{i}\left(S, \mathscr{L} \otimes \mathbb{R}^{j} f_{*}\left(\omega_{X / L}\right)\right)=0
$$

(In fact, as was pointed out to the author by the referee, such vanishing results can also be proven by the same technique as that used in [2] to prove the Kodaira Vanishing Theorem.) When the base $S$ is projective, then Kollar (in [10]) shows how to pass from a vanishing result such as the result of Illusie just quoted to a semi-positivity result such as the one given in Theorem 3.4. On the other hand, if a vector bundle $\mathscr{E}$ on $S$ is found to be semi-positive, and $\mathscr{L}$ is an ample line bundle on $S$, then $\mathscr{F}=\mathscr{E} \otimes_{\mathcal{O}_{S}} \mathscr{L}$ forms an ample vector bundle on $S$. It is not difficult to see that if one applies Kodaira vanishing to the bundle $\mathcal{O}(1)$ on the projective bundle $P \rightarrow S$ associated to $\mathscr{F}$, one then gets a vanishing result similar to the above quoted result of Illusie, but somewhat weaker. Moreover, when $S$ is a curve, it follows immediately from Serre duality that Theorem 3.4 (for the case of trivial coefficients) is equivalent to the vanishing result of Illusie. However, in the case of higher dimensional $S$, especially, when $S$ is not projective, it is not clear to the author what the precise relationship is between Illusie's result quoted above and Theorem 3.4 .

The author would like to express his gratitude to Prof. B. Mazur for 
reading over a preliminary draft of this paper and informing him of the above mentioned paper of Illusie; and to Profs. J. Harris, D. Abramovich, L. Illusie, and the referee for their comments and for pointing out some errors in early versions of this paper. Finally, we thank Prof. A. Ogus for suggesting a significant simplification of the original argument.

\section{§1. Basic Algebraic Lemmas}

Let $A$ be a regular noetherian ring. Let $X, S$ be smooth, proper schemes over $A$, and let $D \subseteq S, E \subseteq X$ be relative divisors with normal crossings over $A$. Let $f: X \rightarrow S$ be a morphism which is smooth over $S-D$, and such that (schematically) $E=f^{-1}(D)$. Note that these assumptions together imply that $f$ is, in fact, smooth in codimension one. To simplify things, we also assume that $f$ has geometrically connected fibres and constant relative dimension. Then, using the terminology of log schemes as given in [8], we make the following

Definition 1.1. We say that $(f: X \rightarrow S ; E ; D)$ (or just $f: X \rightarrow S$, for short) is a semistable family of varieties (or just semistable for short) if, when we give $X$ (respectively, $S$ ) the log structure arising from the divisor with normal crossings $E$ (respectively, $D$ ), the morphism of $\log$ schemes induced by $f$ is log-smooth.

If $f: X \rightarrow S$ is semistable, then we denote the relative sheaf of logarithmic differentials by $\Omega_{X / S}^{\log }$. If the relative dimension of $f$ is $d$, then we denote the $d^{\text {th }}$ exterior power of $\Omega_{X / S}^{\log }$ by $\omega_{X / S}$. Note that $\omega_{X / S}$ is the relative canonical sheaf of $X$ over $S$ in the sense of duality theory.

Proposition 1.2. Suppose that $A$ is an algebraically closed field, that $S$ is one-dimensional (i.e., a curve), and that $\mathscr{L}$ is an ample line bundle on $S$. Then for any $N \in \mathbf{N}$, there exists a finite, flat morphism $\pi: T \rightarrow S$ which has the following properties:

(1) $T$ is a smooth, proper, connected A-scheme;

(2) the ramification divisor $R \subseteq S$ of $\pi$ avoids $D$

(3) $\operatorname{dim}_{A} H^{\mathrm{O}}\left(T, \mathscr{L}_{T}\right) \geq N$.

Proof. This is the standard technique of passing to a ramified cyclic covering, used, for instance, in algebraic proofs of the Kodaira Vanishing Theorem (see [2]). Namely, one takes a very high tensor power of $\mathscr{L}$, say $\mathscr{M}=\mathscr{L}^{\otimes M}$ (where we take $M$ to be very large, and invertible in $A$ ), such that $\mathscr{M}$ is very ample. Then there exists a section $\sigma$ of $\mathscr{M}$ over $S$ whose zero divisor is étale over $A$ and avoids $D$. Then taking the cyclic covering of $S^{\prime}$ 
given by extracting an $M^{\text {th }}$ root of $\sigma$ gives us a scheme $T$ as above such that the dimension of the space of sections over $T$ of $\mathscr{L}$ is one greater than it was before. Repeating this process gives the inequality desired.

Let us go back to assuming that $A$ is arbitrary regular noetherian ring. We now review some basic well-known facts about blow-ups. Suppose that we are given a $A$-flat subscheme $F \subseteq S$. Let us say that $F$ is flatly positioned (respectively, transversely positioned) with respect to the divisor with normal crossings $D$ if for any étale morphism $U \rightarrow S$ such that $\left.D\right|_{U}$ splits into a union of smooth components, the schematic intersection of $\left.F\right|_{U}$ with any irreducible component of $\left.D\right|_{U}$ is flat over $A$ (respectively, and the restriction to any connected component $\left.I \subseteq F\right|_{U}$ of $\left.F\right|_{U}$ of the union of irreducible components of $\left.D\right|_{U}$ that do not contain $I$ is a relative divisor with normal crossings on $I$ ).

Lemma 1.3. Let us suppose that $A$ is a domain, and that we are given a section $\sigma: \operatorname{Spec}(A) \rightarrow S$ whose image is flatly positioned with respect to $D$. Let $\pi: \tilde{S} \rightarrow S$ be the blow-up of $S$ at the image of $\sigma$. Then $\tilde{S}$ is smooth over $A$, and $\tilde{D}=\pi^{-1}(D)_{\text {red }} \subseteq \tilde{S}$ is a relative divisor with normal crossings over $A$.

Proof. Indeed, it suffices to take local coordinates $x_{0}, x_{1}, \ldots, x_{n}$ for $S$ over $A$ at some point in the image of $\sigma$ such that the image of $\sigma$ is defined by the ideal generated by the $x$ 's, and $D$ is defined by some product of the $x$ 's. The result follows by direct computation.

Lemma 1.4. Suppose that $A$ is an algebraically closed field, and that we are given an $A$-smooth curve (i.e., an A-smooth, closed subscheme of dimension one) $C \subseteq S$. Then, after applying a series of blow-ups of $S$ at closed points, we can obtain a birational morphism $\pi: \tilde{S} \rightarrow S$ such that if $\tilde{C}$ is the strict transform of $C$ in $\tilde{S}$, and $\tilde{D}=\pi^{-1}(D)_{\text {red }}$, then $\tilde{C}$ is transversely positioned with respect to $\tilde{D}$.

Proof. Clearly we can assume (by using étale localization) that $D$ splits into a union of smooth divisors: $D=\bigcup D_{i}$. Now we claim that after a series of blow-ups, we may assume that each $D_{i}$ either contains $C$ or intersects $C$ transversely. Indeed, if $D_{i}$ intersects $C$ at a point with multiplicity $r$, then a simple calculation reveals that after blowing up at that point, the strict transform of $D_{i}$ intersects the strict transform of $C$ with multiplicity $\leq r-1$, while the exceptional divisor intersects $C$ transversely. Thus, repeating this process proves the claim.

Thus, we assume that each $D_{i}$ either contains $C$ or intersects $C$ transversely. For simplicity, by localizing, we can also assume that $C$ fails to be transversely positioned with respect to $D$ at only one point. With these assumptions, the only thing that could keep $C$ from being transversely positioned is if two or more distinct $D_{i}$ 's intersect $C$ transversely at the same point. If 
this happens, then let us blow up the point. It is then easy to see that the strict transforms of those $D_{i}$ 's do not meet the strict transform of $C$. This completes the proof.

Now let us go back to assuming that $A$ is a regular noetherian ring. Let us suppose that $C$ is a smooth, geometrically connected curve over $A$ which is embedded as a transversely positioned closed subscheme $C \subseteq S$ with respect to the divisor $D$. Let us denote by $S^{\log }$ the $\log$ scheme (see [8] for details) obtained by putting on $S$ the $\log$ structure associated to the divisor with normal crossings $D$. Let $C^{v i r}$ be the log scheme obtained by placing on $C$ the $\log$ structure obtained by pulling back the $\log$ structure of $S^{\log }$ to $C$. (Here "vir" stands for virtual, since the log structure involves "virtual divisors" that don't really exist on $C$.) Finally, let $C^{\text {phs }}$ be the log scheme obtained by putting on $C$ the $\log$ structure obtained étale locally on $C$ by restricting to $C$ only the components of $D$ that do not contain $C$. (Here "phs" stands for "physical" since the log structure arises from actual "physical" divisors on C.) Thus, we have natural morphisms of $\log$ schemes $C^{\text {vir }} \rightarrow S^{\text {log }}$, and $v: C^{\text {vir }} \rightarrow$ $C^{p h s}$. Note that $v$ induces, via pull-back, a locally split morphism of vector bundles $v^{*}: \Omega_{C^{p h s} / A} \rightarrow \Omega_{C^{v i r} / A}$ on $C$; let us denote the cokernel vector bundle by $\operatorname{Res}_{C^{v i r / A}}$. Thus, intuitively speaking, $\operatorname{Res}_{C^{v i r / A}}$ corresponds to the residues with respect to the components of $D$ that contain $C$. It is easy to see that $\operatorname{Res}_{C^{v i r} / A}$ becomes trivial after pull-back to some finite étale morphism $G \rightarrow C$. Indeed, one need merely take a covering $G \rightarrow C$ on which the étale local system of "smooth irreducible components of $D$ that contain $C$ " splits. On such a $G$, one then obtains a global (i.e., over all of $G$ ) basis for $\operatorname{Res}_{C^{v i r} / A}$ by considering the logarithmic differentials of functions defining the various smooth irreducible components. Thus, in the future, often it will be convenient to assume, in this situation, that $\operatorname{Res}_{C^{v i r} / A}$ already is trivial, and it is always possible to assume this by replacing $C$ by the finite étale cover $G$.

\section{§ 2. Faltings' Extension of Fontaine-Laffaille Theory}

In this Section, we review the definition of Faltings' category $\mathscr{M}_{[a, b]}^{\nabla}\left(S^{\circ}\right)$ of "logarithmic crystalline filtered vector bundles with Frobenius action," and study certain functoriality properties of this category which are not mentioned in [1]. In this Section and throughout the rest of the paper (except the Appendix), we adopt the following conventions: $k$ is a finite field with $q=p^{n}$ elements, $A=W(k)$ (the ring of Witt vectors with residue field $k$ ), and $0<$ $\operatorname{dim}_{A}(S) \leq \operatorname{dim}_{A}(X) \leq p-2$ (where $\operatorname{dim}_{A}(S)$ is the relative dimension of $S$ over A).

Let us choose an affine open $U \subseteq S$, with $U=\operatorname{Spec}(R)$. Let us suppose that we are given an $A$-semilinear (i.e., with respect to the natural Frobenius 
on $A$ ) endomorphism $\Phi: \hat{R} \rightarrow \hat{R}$ of the $p$-adic completion $\hat{R}$ of $R$ that lifts the Frobenius on $R / p R$ and fixes the divisor $D \subseteq S$. Let $a, b \in \mathbb{N}$ with $a \leq b$ and $b-a \leq p-1$. Then by definition ([1], II, c)) an object of $\mathscr{M}_{[a, b]}^{\nabla}\left(R^{\circ}\right)$ consists of the data of a collection of finitely generated $p$-torsion $R$-modules $M, F^{i}(M)$ (for all $i \in \mathbb{Z}$ ), together with an integrable logarithmic connection $\nabla: M \rightarrow$ $M \otimes_{R} \Omega_{R / A}^{l o g}$, plus locally split $R$-linear injections $F^{i+1}(M) \rightarrow F^{i}(M), F^{i}(M) \rightarrow M$, and $R$-linear morphisms $\phi^{i}: F^{i}(M) \otimes_{\hat{R}, \Phi} \hat{R} \rightarrow M$ that satisfy the following axioms:

(1) the composite of $F^{i+1}(M) \rightarrow F^{i}(M)$ with $F^{i}(M) \rightarrow M$ is the morphism $F^{i+1}(M) \rightarrow M$

(2) the morphism $F^{i}(M) \rightarrow M$ is an isomorphism if $i \leq a ; F^{i}(M)=0$ if $i>b$;

(3) if we let $\tilde{M}$ be the inductive limit of the diagram:

$$
\cdots \rightarrow F^{i+1}(M) \leftarrow F^{i+1}(M) \rightarrow F^{i}(M) \leftarrow F^{i}(M) \rightarrow F^{i-1}(M) \leftarrow \cdots
$$

where the right arrows are the morphisms in the definition and the left arrows are multiplication by $p$, then we assume that the $\phi^{i}$ induce an isomorphism $\phi: \tilde{M} \otimes_{\hat{R}, \Phi} \hat{R} \rightarrow M$; (thus, here we require that $\left.\phi^{i-1}\right|_{F^{i}(M)}=p \phi^{i}$;)

(4) the connection $\nabla$ satisfies Griffiths transversality, i.e., $\nabla\left(F^{i}(M)\right) \subseteq$ $F^{i-1}(M) \otimes_{R} \Omega_{R / A}^{\log }$

(5) the isomorphism $\phi: \tilde{M} \otimes_{\hat{R}, \Phi} \hat{R} \rightarrow M$ are parallel with respect to the connection $\nabla$ on $M$, and the connection naturally induced by $\nabla$ on $\tilde{M} \otimes_{\hat{R}, \Phi} \hat{R}$ (as in [1], §2).

Although this local definition depends on the choice of our Frobenius lifting $\Phi$, it is possible to prove ([1], Theorem 2.3-which holds since we are assuming that $p>2$ ) that for any two choices of $\Phi$, there is a canonical isomorphism between the two resulting $\mathscr{M}^{\mathscr{F}_{[a, b]}} \nabla\left(R^{\circ}\right)^{\prime}$. Thus, we can glue these local categories together and thus obtain a global category, which is denoted $\mathscr{M}_{[a, b]}^{\nabla}\left(S^{\circ}\right)$.

One natural way to obtain objects in $\mathscr{M}_{[a, b]}^{\nabla}\left(S^{\circ}\right)$ is by taking the higher direct image sheaves with respect to $f_{c r}^{\circ}$ of logarithmic crystals on $X$. Indeed, we may rephrase this statement in greater detail as follows. Let $\mathscr{E}$ be an object of $\mathscr{M}_{[0, a]}^{\nabla}\left(X^{\circ}\right)$. Now by regarding $X$ and $S$ as being endowed with logarithmic structures arising from the divisors $E$ and $D$, we may consider (following [8], §4) the relative crystalline sites of $X$ and $S$ (with their respective $\log$ structures) over $A$. Since $\mathscr{E}$ is endowed, in particular, with a connection, it defines a crystal on the relative crystalline site of $X^{\circ}$ over $A$. Thus, we 
can take the higher direct image sheaves $\mathbf{R}^{i}\left(f_{c r}^{\circ}\right)_{*} \mathscr{E}$ with respect to the morphism of topoi $f_{c r}^{\circ}$ from the logarithmic crystalline site of $X$ to that of $S$. It is then a result of [1], Theorem 6.2 , that these higher direct images $\mathbf{R}^{i}\left(f_{c r}^{\circ}\right)_{*} \mathscr{E}$ come with a natural filtration and Frobenius action in such a way that we may regard $\mathbf{R}^{i}\left(f_{c r}^{\circ}\right)_{*} \mathscr{E}$ as being an object of $\mathscr{M}_{[0, a+i]}^{\nabla}\left(S^{\circ}\right)$, as long as $\max (a+d$, $a+i) \leq p-2$. A typical example is the case where $\mathscr{E}$ is taken to be $\mathcal{O}_{\boldsymbol{X}_{n}}=$ $\mathcal{O}_{X} \otimes_{\mathbf{z}_{p}} \mathbf{Z} / p^{n} \mathbf{Z}$ (with the obvious "trivial" filtration, connection, and Frobenius action), and $a=0$.

Later, following a suggestion of Ogus (to simplify the original proof) we will make use of the following:

Lemma 2.1. Let $\left(M, F^{i}(M), \nabla, \phi^{i}\right)$ be an object of $\mathscr{M}_{[0, a]}^{\nabla}\left(S^{\circ}\right)$, where $S$ is smooth and proper, and $a<p-2$. Then every element of $H^{0}\left(S, M / F^{1}(M)\right)$ lifts to a horizontal element of $H^{0}(S, M)$.

Proof. This follows from the degeneration of the Hodge spectral sequence ([1], Theorem 4.1).

This completes our review of the definition of $\mathscr{M}_{[a, b]}^{\nabla}\left(S^{\circ}\right)$. We now wish to discuss certain functoriality properties of the category $\mathscr{M}_{[a, b]}^{\nabla}$. Let us assume that we are given an $A$-smooth closed, one-dimensional subscheme $C \subseteq S$ which is transversely positioned (see $\S 1$ for a definition) with respect to $D$. Let $F \subseteq C$ be the divisor (necessarily étale over $A$ ) obtained by restricting to $C$ the irreducible components of $D$ that do not contain $C$. (In fact, these irreducible components may only exist after étale localization, so one may need to apply étale descent to construct $F$, but this is no problem.) With the choice of divisor $F \subseteq C$, we may consider the category $\mathscr{M}_{[a, b]}^{\nabla}\left(C^{\circ}\right)$. We wish to construct a restriction functor

$$
\rho: \mathscr{M F}_{[a, b]}^{\nabla}\left(S^{\circ}\right) \rightarrow \mathscr{M}_{[a, b]}^{\nabla}\left(C^{\circ}\right)
$$

as follows. Clearly, we can use étale descent, and thus assume that $S=$ $\operatorname{Spec}(R), C=\operatorname{Spec}(T)$ and that $D$ splits as a union of smooth divisors. Also, we use the notation of "vir" and "phs" to denote the various log structures on $C$ defined at the end of $\S 1$. We start with $R$-modules $M, F^{i}(M)$, together with various split injections among these modules and an integrable logarithmic connection $\nabla: M \rightarrow M \otimes_{R} \Omega_{R / A}^{\log }$. These restrict to modules $M_{C}, F^{i}(M)_{C}$, along with various split injections and an integrable logarithmic connection $\nabla_{C}^{\text {vir: }}$ $M_{C} \rightarrow M_{C} \otimes_{T} \Omega_{C^{v i r} / A}^{\log _{A}}$. Of course, ideally, we would like an object with connection on the $\log$ scheme $C^{\text {phs }}$, not $C^{\text {vir. }}$. To obtain such an object we reason as follows. First, by (finite) étale descent, we assume that $\operatorname{Res}_{\mathrm{C}^{v i r} / A}$ is a trivial vector bundle, and we choose a basis of generating sections of $\operatorname{Res}_{C^{v i r / A}}$ over $C$ each of which is locally obtained as the logarithmic differential of a function defining a smooth irreducible component of $D$, and we let $s_{1}, \ldots, s_{r}$ be the 
corresponding basis of the dual vector bundle $\operatorname{Res}_{C^{v i r} / A}^{v}$. By applying $\nabla_{C}^{v i r}$ to each of the $s_{i}$, we obtain a collection of commuting (because of the integrability of $\nabla$ ) $\mathcal{O}_{C}$-linear endomorphisms $N_{i}: M_{C} \rightarrow M_{C}$, for $i=1, \ldots, r$. Moreover, since they represent the monodromy of an object of $\mathscr{M}_{[a, b]}^{\nabla}$, the $N_{i}$ are necessarily nilpotent. Indeed, to see this, we reason as follows: Let $N$ be one of the $N_{i}$ 's. Note that there exists (by condition (3) above) a "Verschiebung" morphism $V: M \rightarrow M \Theta_{\hat{R}, \Phi} \hat{R}$ such that $\phi^{a} \circ V=p^{j}$ and $V \circ \phi^{a}=p^{j}$, where $j \stackrel{\text { def }}{=}$ $b-a$. Thus, (by condition (5) above) $N^{j}=\phi^{a} \circ N^{j} \circ V$, so $N^{2 j}=p^{j} \cdot \phi^{a} \circ N^{2 j} \circ$ $V$. Thus, by recursion, $N^{2 j}=0$. This proves that the $N_{i}$ are nilpotent.

Thus, the $N_{i}$ define a filtration with $r$ indices, which we call the monodromy filtration on $M_{C}$, and which we denote by:

$$
\mu_{i_{1}, \ldots, i_{\alpha}, \ldots, i_{r}}\left(M_{C}\right)=\operatorname{Ker}\left(N_{1}^{i_{1}} \ldots N_{\alpha}^{i_{\alpha}} \ldots N_{r}^{i_{r}}\right) .
$$

Now let $G r_{\mu}\left(M_{C}\right)$ denote the graded object associated to the monodromy filtration. It is clear that the filtration $F^{i}(M)_{C}$ defines a natural filtration $F^{i}\left(G r_{\mu}\left(M_{C}\right)\right)$ on $G r_{\mu}\left(M_{C}\right)$, and that $\nabla_{C}^{v i r}$ induces a connection $\nabla^{g r}: G r_{\mu}\left(M_{C}\right) \rightarrow$ $G r_{\mu}\left(M_{C}\right) \otimes_{T} \Omega_{C^{p h s / A}}^{\log }$, as desired. It is easy to see that $\left(G r_{\mu}\left(M_{C}\right), F^{i}\left(G r_{\mu}\left(M_{C}\right)\right), \nabla^{g r}\right)$ satisfy all the necessary axioms for an object of $\mathscr{M}_{[a, b]}^{\nabla}\left(C^{\circ}\right)$, and all that remains to do in defining $\rho$ is the construction of the Frobenius action on $G r_{\mu}\left(M_{C}\right)$

First of all, because of our assumption that $C$ is transversely positioned, one sees easily that (after possible further étale localization) it is possible to choose a Frobenius lifting $\Phi: \hat{R} \rightarrow \hat{R}$ that not only fixes $D$, but also fixes $C$ (and $F$ ). Thus, $\Phi$ restricts to a Frobenius lifting $\Phi_{C}: \hat{T} \rightarrow \hat{T}$, so that tensoring $\phi$ over $R$ with $T$ (via the natural projection $R \rightarrow T$ ) gives an isomorphism $\phi_{C}: \tilde{M}_{C} \otimes_{T, \Phi_{C}} T \cong\left(\tilde{M} \otimes_{R, \Phi} R\right) \otimes_{R} T \rightarrow M_{C}$. Since this Frobenius action on $M_{C}$ clearly commutes with the $N_{i}$, it follows that the Frobenius action on $M_{C}$ induces a Frobenius action on $G r_{\mu}\left(M_{C}\right)$. One checks easily that the Frobenius that we obtain on $\operatorname{Gr}_{\mu}\left(M_{C}\right)$ is independent of the choice of $\Phi$ (as long as we deal with $\Phi$ that fix $C$ and $D$ ): indeed, the morphism $\alpha$ constructed in the proof of [1], Theorem 2.3, is easily seen to commute with restriction to C. Finally, it is easy to check that this Frobenius action satisfies all the remaining properties in the definition of the category $\mathscr{M}^{\nabla}{ }^{\nabla}$ : Indeed, this follows from using the fact that $\mathscr{M} \mathscr{F}$ (i.e., the category of $[1], \S 2$, that is similar to $\mathscr{M}^{\nabla}$, but lacks the datum of a connection) is abelian ([1], Theorem 2.1, Corollary). This completes the definition of the functor $\rho$.

Now we wish to note that functoriality also holds for blow-ups $\pi: \tilde{S} \rightarrow S$ at a section $\sigma: \operatorname{Spec}(A) \rightarrow S$ which is flatly positioned. The procedure is the same as in the previous paragraph: one pulls back the necessary modules, morphisms, and connection in the obvious fashion; the only trick is pulling back the Frobenius action. The key is to note that since $\sigma$ is flatly positioned, 
one can choose the Frobenius lifting $\Phi: \hat{R} \rightarrow \hat{R}$ in such a way that not only $D$, but also $\sigma$ is fixed by $\Phi$. It is then easy to see that the Frobenius action on $\hat{R}$ extends to a Frobenius action on the $p$-adic completion of $\tilde{S}$ in such a way as to be functorial with respect to $\pi$. It remains to check that $\pi^{*}$ is independent of the choice of Frobenius lifting $\Phi$. But the only thing which is unclear is whether or not the Frobenius action depends on $\Phi$, and this follows from restricting to a dense open subset of $S$ over which $\pi$ is an isomorphism. Thus, we see that we get a natural functor

$$
\pi^{*}: \mathscr{M}_{[a, b]}^{\nabla}\left(S^{\circ}\right) \rightarrow \mathscr{M}_{[a, b]}^{\nabla}\left((\widetilde{S})^{\circ}\right)
$$

as desired.

Now let $C \rightarrow \operatorname{Spec}(A)$ be a proper, smooth curve, with geometrically connected fibres, and let $F \subseteq C$ be an étale divisor over $A$. Let us suppose that we are given a finite morphism $\psi: C \rightarrow S$. Then we shall say that $\psi$ is admissibly factorizable if it factors into the composite of the following form:

(1) we have a morphism $\psi_{1}: \tilde{S} \rightarrow S$, which is the composite of a sequence of blow-ups at $A$-sections that are flatly positioned over $A$; let $\widetilde{D}=$ $\psi_{1}^{-1}(D)_{\text {red }}$;

(2) for some $C^{\prime} \rightarrow \operatorname{Spec}(A)$ (also a smooth curve with geometrically connected fibres) and some $F^{\prime} \subseteq C^{\prime}$ (an étale divisor over $A$ ), we have a closed immersion $\psi_{2}: C^{\prime} \hookrightarrow \tilde{S}$ such that $C^{\prime}$ is transversely positioned with respect to $\tilde{D}$, and $F^{\prime}$ is the restriction to $C^{\prime}$ of the components of $\tilde{D}$ that do not contain $C^{\prime}$; we also assume that $C^{\prime}$ is not contained in any of the exceptional divisors of the morphism $\psi_{1}$;

(3) we have a morphism $\psi_{3}: C \rightarrow C^{\prime}$ such that $\psi_{3}^{-1}\left(F^{\prime}\right)_{\text {red }} \subseteq F$ and $\psi_{3}$ is étale outside of $F$ with tame ramification at $F$;

so that $\psi=\psi_{1} \circ \psi_{2} \circ \psi_{3}$.

Let us suppose that $\psi$ is admissibly factorizable. Then by the functoriality considerations reviewed above, it is clear that by composition we obtain a functor

$$
\psi^{*}: \mathscr{M}_{[a, b]}^{\nabla}\left(S^{\circ}\right) \rightarrow \mathscr{M F}_{[a, b]}^{\nabla}\left(C^{\circ}\right) .
$$

(Indeed, only for morphisms like $\psi_{3}$ did we not discuss functoriality; but functoriality for morphisms of this type is the most obvious.) It remains to see that $\psi^{*}$ is independent of the factorization $\psi=\psi_{1} \circ \psi_{2} \circ \psi_{3}$. This is clear for all the data involved except (perhaps) for the Frobenius action and the formation of $\mathrm{Gr}_{\mu}(-)$; but this follows, as usual, by restricting to the open dense subset $C-F$, where the fact that the Frobenius action and the formation 
of $G r_{\mu}(-)$ are independent of the factorization is clear. We thus summarize our findings in the following

Proposition 2.2. For all admissibly factorizable morphisms $\psi:(C, F) \rightarrow$ $(S, D)$, we have a canonical functor

$$
\psi^{*}: \mathscr{M}_{[a, b]}^{\nabla}\left(S^{\circ}\right) \rightarrow \mathscr{M}_{[a, b]}^{\nabla}\left(C^{\circ}\right)
$$

for all $b-a \leq p-1$.

\section{§3. The Main Theorem}

Before stating and proving the Main Theorem of this paper, we need to introduce some new definitions. Unfortunately, these definitions are somewhat ad hoc, but at least they allow us to prove a theorem with a somewhat broader range of applicability than previous statements of such results (as given in [5], [9]).

Let $L$ be a field of characteristic zero. Let $X$ be a proper, smooth $L$-scheme; $E \subseteq X$ a divisor with normal crossings. Fix $a \in \mathbf{N}$. Let us suppose that we are given a vector bundle $\mathscr{A}$ on $X$ together with a filtration $F^{a}(\mathscr{A}) \subseteq$ $F^{a-1}(\mathscr{A}) \subseteq \cdots F^{1}(\mathscr{A}) \subseteq F^{0}(\mathscr{A})=\mathscr{A}$ by subbundles such that all the inclusions are locally split, and a connection $\nabla_{\mathscr{A}}$ with logarithmic poles at $E$. Then we make the following

Definition 3.1. (i) Suppose that $L$ is a number field (i.e., a finite extension of $Q$ ). Let $\mathcal{O}_{L}$ be its ring of integers. Then for some positive rational integer $N$, the objects $X, E, \mathscr{A}$, and $\nabla_{\mathscr{A}}$ are defined over $R \stackrel{\text { def }}{=} \mathcal{O}_{L}\left[N^{-1}\right]$ and satisfy the same hypotheses as they did over $L$ (i.e. being proper and smooth, being a relative divisor with normal crossings, being a vector bundle, being a locally split inclusion, being a connection with logarithmic poles at $E$, etc.). Let us fix such models $X_{R}, E_{R}, \mathscr{A}_{R}$, and $\left(\nabla_{\mathscr{A}}\right)_{R}$ over $R$, and denote by means of a subscript the result of base-changing these objects from $R$ to some $R$-algebra. Then we shall say that $\left(\mathscr{A}, \nabla_{\mathscr{A}}, F^{i}(\mathscr{A})\right)$ is globally crystalline if for almost all primes $\wp$ of $L$, when we base-change from $R$ to the completion $A_{\wp}$ of $\mathcal{O}_{L}$ (the ring of integers) at $\wp$, the resulting filtered vector bundle with connection arises from an inverse system of objects $\left\{\mathscr{A}_{\wp, n}\right\}_{n \in \mathbb{N}}$ (where $\mathscr{A}_{\wp, n}$ is flat over $\left.\mathbb{Z} / p^{n} \mathbb{Z}\right)$ of $\mathscr{M}_{[0, a]}^{\nabla}\left(X_{\mathscr{A}_{\wp}}^{\circ}\right)$.

(ii) If $L$ is any field (of characteristic zero), then we say that $\left(\mathscr{A}, \nabla_{\mathscr{A}}, F^{i}(\mathscr{A})\right.$ ) is globally crystalline if there is a finitely generated $\mathbf{Q}$-subalgebra $R \subseteq L$ over which $X, E, \mathscr{A}, \nabla_{\mathscr{A}}$, and the $F^{i}(\mathscr{A})$ are defined and satisfy the same hypotheses as they did over $L$ (i.e. being proper and smooth, being a relative divisor with normal crossings, being a vector bundle, being a locally split inclusion, being a connection with logarithmic poles at $E$, etc.) such that at all closed 
points of Spec $(R)$ (whose residue fields and necessarily number fields), the restricted filtered vector bundle is globally crystalline (as in (i)).

Note that if $\left(\mathscr{A}, \nabla_{\mathscr{A}}, F^{i}(\mathscr{A})\right)$ is globally crystalline, then the $\mathcal{O}_{X}$-dual of $\mathscr{A}$, which we denote by $\mathscr{A}^{\vee}$, endowed with the dual filtration (shifted over $a$ places) and connection, gives rise to a filtered vector bundle with connection $\left(\mathscr{A}^{\vee}, \nabla_{\mathscr{A}^{v}}, F^{i}\left(\mathscr{A}^{\vee}\right)\right)$ which is again globally crystalline. Continuing with our field $L$ of characteristic zero, suppose that $X$ is proper and geometrically connected, and that we are also given a smooth, proper, geometrically connected $L$-scheme $S$ and a divisor with normal crossings $D \subseteq S$, plus a morphism $f:(X, E) \rightarrow(S, D)$ which is semistable in the sense of Definition 1.1.

Lemma 3.2. We denote by $d$ the relative dimension of $X$ over $S$. Suppose that $\left(\mathscr{A}, \nabla_{\mathscr{A}}, F^{i}(\mathscr{A})\right)$ is globally crystalline. Then for any $i \geq 0$, the de Rham cohomology $\mathbf{R}^{d-i}\left(f_{D R}^{\circ}\right)_{*}\left(\mathscr{A}, \nabla_{\mathscr{A}}\right)$, together with the Gauss-Manin connection, and the Hodge filtration (induced by $F^{i}(\mathscr{A})$ ) constitute a globally crystalline object on $(S, D)$. Let $\left(\mathscr{B}, \nabla_{\mathscr{B}}, F^{i}(\mathscr{B})\right)$ be the dual object to this crystalline object on $(S, D)$. Then,

$$
\left(\mathscr{B} / F^{1}(\mathscr{B})\right)^{\vee}=\mathbb{R}^{i} f_{*}\left(\omega_{X / S} \otimes_{\mathcal{O}_{X}}\left(\mathscr{A} / F^{1}(\mathscr{A})\right)^{\vee}\right) .
$$

Proof. This follows from [1], Theorem 6.2, and Grothendieck-Serre duality applied to the morphism $f$ (for which $\omega_{X / S}$ is the dualizing sheaf).

Lemma 3.3. Assume that $L$ is algebraically closed. Suppose that we have a vector bundle with connection $\left(\mathscr{A}, \nabla_{\mathscr{A}}\right)$ on a $X$ with logarithmic poles at $E$. Then $\operatorname{dim}_{L} H_{D R}^{0}(X, \mathscr{A}) \leq \operatorname{rank}(\mathscr{A})$, where by the subscript "DR," we mean the horizontal global sections.

Proof. By formal integration using the connection, and the fact that in characteristic zero, the exterior derivative vanishes only on constant functions, it follows that a horizontal global section is determined by its value at a single point.

We now review some basic definitions about semi-positivity from [5]. Let $V$ be a projective variety over a field $L$. Then a line bundle $\mathscr{L}$ on $V$ is semi-positive if for any finite morphism $g: C \rightarrow V$, where $C$ is a smooth, projective curve, the degree of $g^{*} \mathscr{L}$ is nonnegative. If $\mathscr{E}$ is a vector bundle on $V$, then $\mathscr{E}$ is semi-positive if the $\mathcal{O}(1)$-bundle on $\mathbf{P}_{V}(\mathscr{E})$ is semi-positive. It follows immediately from the definitions that if $\mathscr{E}$ is semi-positive and $\mathscr{L}$ is an ample line bundle, then $\mathscr{E} \otimes_{\mathcal{O}_{V}} \mathscr{L}$ is an ample vector bundle on $V$.

We are now ready to state the Main Theorem of this paper. Various forms of this theorem were proven previously by Fujita ([5]) and Kawamata ([9]). Moreover, as Viehweg remarks in [11], $\$ 1.21$, the techniques of $\mathbf{J}$. Kollar, when taken with the results of Deligne on the degeneration of the 
Hodge-Deligne spectral sequence, constitute an algebraic proof of this sort of result. Strictly speaking, the result given here does not quite imply Kawamata's result because our definition of semistability (involving a geometric condition on the divisors at infinity, as opposed to a monodromy-type condition) is stronger than Kawamata's. (Kollar uses a similar definition to our own.) Finally, as mentioned in the Introduction, there is the paper [7]. The proof given here is a simplification of the author's original proof, following a suggestion of Prof A. Ogus.

Theorem 3.4. Let $f:(X, E) \rightarrow(S, D)$ be a semistable family of varieties of relative dimension $d$, with $S$ a smooth, proper scheme over a field $L$ of characteristic zero. Let $\left(\mathscr{A}, \nabla_{\mathscr{A}}, F^{i}(\mathscr{A})\right)$ be a globally crystalline filtered vector bundle with connection on $(X, E)$. Then for any nonnegative integer $\alpha$, the coherent sheaf of $\mathcal{O}_{S}$-modules

$$
\mathbf{R}^{\alpha} f_{*}\left(\omega_{X / S} \otimes_{\mathcal{O}_{X}}\left(\mathscr{A} / F^{1}(\mathscr{A})\right)^{\vee}\right)
$$

is a semi-positive vector bundle, as are all of its tensor powers.

Proof. First of all, by Lemma 3.2, since taking de Rham cohomology and tensor products does not take us out of the category $\mathscr{M F}^{\nabla}$, we may assume that $f$ is the identity, and we may prove the result for the "first tensor power," i.e., the original globally crystalline object. Also, without loss of generality, let us assume that $L$ is algebraically closed. Let $\mathscr{S}$ be the vector bundle $\left(\mathscr{A} / F^{1}(\mathscr{A})\right)^{\vee}$. Let $r=\operatorname{rank}(\mathscr{A})$.

Let us assume that $\mathscr{S}$ is not semi-positive. Then there exists some smooth, proper, connected curve $C$ over $L$, together with a finite morphism $\psi: C \rightarrow S$ and a nonzero morphism $\mathscr{S}_{C} \rightarrow \mathscr{Q}$, where $\mathscr{Q}$ is a line bundle of negative degree. By Proposition 1.2, after replacing $C$ by a finite covering of $C$, we may assume that $h^{0}\left(C, \mathscr{Q}^{-1}\right) \geq 1+r$. Thus, by dualizing the morphism $\mathscr{I}_{C} \rightarrow$ 2 , we see that $h^{0}\left(C, \mathscr{S}_{C} v \geq 1+r\right.$. On the other hand, by Lemma 1.4 , there exists a birational map $\pi: \tilde{S} \rightarrow S$ obtained as a sequence of blow-ups at closed points such that the morphism $C \rightarrow \tilde{S}$ (obtained from $\psi$ by the valuative criterion of properness) factors through a smooth, proper, connected curve $C^{\prime}$ embedded in $\tilde{S}$ : that is, we have a finite morphism $\psi_{1}: C \rightarrow C^{\prime}$ and an embedding $\psi_{2}: C^{\prime} \hookrightarrow \tilde{S}$ whose composite is the morphism $C \rightarrow \widetilde{S}$. Moreover, by Lemma 1.4, we can assume that the image of $C^{\prime}$ is transversely positioned with respect to $\tilde{D}=\pi^{-1}(D)_{\text {red }}$. It thus follows that by spreading out, specializing, completing, we obtain objects $C_{A}, S_{A}, D_{A}$, etc. over a $\operatorname{ring} A$ satisfying the hypotheses given at the beginning of $\S 2$, together with a divisor $F_{A} \subseteq C_{A}$ such that $\psi_{A}:\left(C_{A}, F_{A}\right) \rightarrow\left(S_{A}, D_{A}\right)$ is admissibly factorizable (in the sense of the definition given directly before Proposition 2.2). Thus, for the rest of the proof, we shall work over $A$, and omit the subscripts for all the objects that have been base changed into objects over $A$. 
Thus, in summary, we have an admissibly factorizable morphism $\psi$ : $(C, F) \rightarrow(S, D)$, such that $h^{0}\left(C_{K}, \mathscr{S}_{C_{K}}^{v}\right) \geq 1+r$, where $K$, as usual, is the quotient field of $A$. Now recall that $\mathscr{S}^{v}=\mathscr{A} / F^{1}(\mathscr{A})$. Let us consider the inverse system $\left(M, \nabla_{M}, F^{i}(M), \phi^{i}\right)$ of objects of $\mathscr{M}_{[0, a]}^{\nabla}\left(S^{\circ}\right)$ associated to $\left(\mathscr{A}, \nabla_{\mathscr{A}}, F^{j}(\mathscr{A})\right)$ whose existence is guaranteed by the fact that $\left(\mathscr{A}, \nabla_{\mathscr{A}}, F^{j}(\mathscr{A})\right)$ is globally crystalline. (We shall regard this inverse system as a single object, which is flat over $\mathbf{Z}_{p}$.) Let us apply the pull-back functor of Proposition 2.2 to $\left(M, \nabla_{M}, F^{i}(M), \phi^{i}\right)$ to obtain a $\mathbb{Z}_{p}$-flat object (actually, an inverse system) $\left(N, \nabla_{N}\right.$, $\left.F^{i}(N), \psi^{i}\right)$ of $\mathscr{M}_{\left.{ }^{0}, a\right]}^{\nabla}\left(C^{\circ}\right)$. Now since $h^{0}\left(C_{K}, \mathscr{S}_{C_{K}}^{v}\right) \geq 1+r$, and $N / F^{1}(N)$ is a direct sum of subquotients of the monodromy filtration on $\mathscr{S}_{C}^{v}=\psi^{*}\left(M / F^{1}(M)\right)$, we thus obtain that

$$
h^{0}\left(C_{K},\left(N / F^{1}(N)\right)_{K}\right) \geq 1+r .
$$

By Lemma 2.1, this implies that $\operatorname{dim}_{K} H_{D R}^{0}\left(C_{K}, N_{K}\right) \geq 1+r$. Since $r$ is the rank of the vector bundle $M$, and hence also of the vector bundle $N$, we thus conclude from Lemma 3.3 that we have a contradiction. This completes the proof.

In particular, let us consider (under the hypotheses of Theorem 3.4) the globally crystalline triple $\left(\mathscr{B}, F^{i}(\mathscr{B}), \nabla_{\mathscr{B}}\right)$ (on $(S, D)$ ) given by $\mathbf{R}^{2 d-i}\left(f_{D R}^{\circ}\right)_{*}\left(\mathcal{O}_{X}\right)$, together with its Hodge filtration and the Gauss-Manin connection. Let $\left(\mathscr{A}, F^{i}(\mathscr{A}), \nabla_{\mathscr{A}}\right)$ be the triple obtained from $\left(\mathscr{B}, F^{i}(\mathscr{B}), \nabla_{\mathscr{B}}\right)$ by shifting the filtration down by $d-i$ places. Just as in Lemma 3.2 , we see that

$$
\left(\mathscr{A} / F^{1}(\mathscr{A})\right)^{\vee}=\left(\mathbb{R}^{d} f_{*}\left(\Omega_{X / S}^{\log }\right)^{d-i}\right)^{\vee}=f_{*}\left(\left(\Omega_{X / S}^{l o g}\right)^{i}\right) .
$$

Thus, if we apply Theorem 3.4 to $\left(\mathscr{A}, F^{i}(\mathscr{A}), \nabla_{\mathscr{A}}\right)$ and the identity morphism on $S$, we see obtain the following:

Corollary 3.5. Let $f:(X, E) \rightarrow(S, D)$ be as above. Then for any nonnegative integer $\alpha$, the coherent sheaf of $\mathcal{O}_{S}$-modules

$$
f_{*}\left(\left(\Omega_{X / S}^{\log }\right)^{\alpha}\right)
$$

is a semi-positive vector bundle, as are all of its tensor powers.

\section{Appendix: The Case of One-Dimensional Fibres}

Let $f: X \rightarrow S, D$, and $E$ be as in $\S 1$, with $f$ semistable and of relative dimension one, and suppose that $S$ is a curve. Let us assume that the ring $A$ in $\S 1$ is an algebraically closed field $k$ of characteristic zero. Then we have the following

Theorem. The vector bundle $f_{*} \omega_{X / S}$ on $S$ is semi-positive in the sense that there do not exist nonzero morphisms $\phi: f_{*} \omega_{X / S} \rightarrow \mathscr{Q}$, where 2 is a line bundle on $S$ of negative degree. 
Proof. Let us assume that such a $\phi$ exists. By Proposition 1.2, we may assume that $\operatorname{dim}_{k} H^{0}\left(S, \mathscr{Q}^{-1}\right) \geq g+1$, where $g$ is the genus of the generic fibre of $f$. Thus, by considering $\phi^{\vee}: \mathscr{Q}^{-1} \rightarrow \mathbb{R}^{1} f_{*} \mathcal{O}_{X}$, we see that $\operatorname{dim}_{k} H^{0}\left(S, \mathbb{R}^{1} f_{*} \mathcal{O}_{X}\right) \geq$ $g+1$. By the Leray-Serre spectral sequence for $f$ applied to the sheaf $\mathcal{O}_{X}$, we see that we have, in fact, $\operatorname{dim}_{k} H^{1}\left(X, \mathcal{O}_{X}\right) \geq q+g+1$, where $q$ is the genus of $S$. On the other hand, since we are in characteristic zero, $\operatorname{dim}_{k} H^{1}\left(X, \mathcal{O}_{X}\right)$ is exactly the dimension of $\operatorname{Pic}^{0}(X)$ over $k$. Thus, we see that $\operatorname{dim}_{k}\left(\operatorname{Pic}^{0}(X)\right) \geq$ $q+g+1$.

Now we recompute the dimension of $\operatorname{Pic}^{0}(X)$ by means of $l$-adic cohomo$\log$ to see that this is absurd. Indeed, by applying the Leray-Serre spectral sequence for $f_{e t}$ to the étale sheaf $\mathbb{Q}_{l}(1)$, we see that $\operatorname{dim}_{\mathbf{Q}_{1}} H^{1}\left(X_{e t}, \mathbb{Q}_{l}(1)\right) \leq$ $2 q+2 g$. On the other hand, Kummer theory tells us that $\operatorname{dim}_{\mathbb{Q}_{l}} H^{1}\left(X_{e t}, \mathbb{Q}_{l}(1)\right)$ is equal to twice the dimension (over $k$ ) of $\operatorname{Pic}^{0}(X)$ (since $\operatorname{Pic}^{0}(X)$ is an abelian variety over $k$ ). Putting this together with the inequality derived in the first paragraph, we obtain a contradiction.

\section{Bibliography}

[1] Faltings, G., Crystalline Cohomology and p-adic Galois Representations, Proceedings of the First JAMI Conference, Johns Hopkins University Press (1990), 25-79.

[2] - p-adic Hodge Theory, J. AMS, 1 (1988), 255-299.

[3] Fontaine, J. M. and Laffaille, G., Construction de représentations p-adiques, Ann. Sci. ENS., 15 (1982), 547-608.

[4] Fontaine, J. M., Sur certains types de représentations $p$-adiques du groupe de Galois d'un corps local: construction d'un anneau de Barsotti-Tate, Ann. of Math., 115 (1982), 529-577.

[ 5 ] Fujita, T., On Kähler Fibre Spaces over Curves, J. Math. Soc. Japan, 30 (1978), 779-794.

[6] Hartshorne, R., Algebraic Geometry, Graduate Texts in Mathematics 52, Springer-Verlag, 1977.

[7] Illusie, L., Réduction semi-stable et décomposition de complexes de de Rham à coefficients, Duke Math. J., 60, No. 1 (1990), 139-185.

[ 8 ] Kato, K., Logarithmic Structure of Fontaine-Illusie, Proceedings of the First JAMI Conference, Johns Hopkins University Press (1990), 191-224.

[9] Kawamata, Y., Characterization of Abelian Varieties, Compositio Math., 43 (1981), 253276.

[10] Kollar, J., Higher Direct Image Sheaves I, Ann. of Math., 123 (1986), 11-42.

[11] Viehweg, E., Weak Positivity and the Stability of Certain Hilbert Points, Invent. Math., 96 (1989), 639-667. 